\title{
Recent advances in speckle decorrelation modeling and processing in digital holographic interferometry
}

\author{
Pascal Picart ${ }^{* 1,2}$ \\ ${ }^{1}$ Laboratoire d'Acoustique de l'Université du Mans, CNRS 6613, Institut d'Acoustique - Graduate School (IA-GS), \\ Le Mans Université, Avenue Olivier Messiaen, 72085 Le Mans Cedex 9, France \\ ${ }^{2}$ École Nationale Supérieure d'Ingénieurs du Mans, rue Aristote, 72085 Le Mans Cedex 9, France
}

Received November 16, 2021; accepted December 23, 2021; published December 31, 2021

\begin{abstract}
Digital holography, and especially digital holographic interferometry, is a powerful approach for the characterization of modifications at the surface or in the volume of objects. Nevertheless, the reconstructed phase data from holographic interferometry is corrupted by the speckle noise. In this paper, we discuss on recent advances in speckle decorrelation noise removal. Two main topics are considered. The first one presents recent results in modelling the decorrelation noise in digital Fresnel holography. Especially the anisotropy of the decorrelation noise is established. The second topic presents a new approach for speckle de-noising using deep convolution neural networks.
\end{abstract}

Digital holography is a powerful approach for remote metrology at different scales (micro and macro) [1]. Holographic interferometry provides an optical path difference in the wrapped modulo $2 \pi$ phase. The phase is connected to the scene/object/structure of interest and can be helpfully considered for many industrial purposes: roughness measurements [2], surface shape profiling [3], surface deformation [4] or vibration measurements [5]. Holographic interferometry has the benefit of being contact-less and provides full-field measurements. In addition, the use of light illumination makes it nonintrusive. High temporal resolution can be obtained when using high-speed cameras [5]. For example, holographic interferometry is adapted for the investigation of fundamental properties of transient mechanical waves propagating in complex metamaterials [6]. The use of long wavelength infrared radiations enables large deformation measurements, which are of interest for extended structures [7], by providing desensitization pf holographic measurements by a factor of almost 20 [4].

From the practical point of view, the change in the optical phase from digitally reconstructed holograms is of interest and is obtained within modulo of $2 \pi$. Basically, the speckle pattern produced from the object surface under coherent illumination is modified as well as changes from its initial state. It follows that the phase from holograms is also speckled. Therefore, speckle decorrelation noise is included in phase changes and advanced filtering is required to get noise-free phase maps [8-9]. Recently, noteworthy progresses in the

*E-mail: pascal.picart@univ-lemans.fr understanding and modeling of the image-to-object relationship in holographic imaging has been obtained [1]-[12]. However, digital holographic interferometry is still limited by the requirement of fast data processing. For that, two main directions must be investigated: first, full modelling of speckle decorrelation noise and last, artificial intelligence (AI) [10] as a new paradigm to boost the computation time in digital holographic data processing.

Recently, we have addressed the first point and proposed modelling of speckle decorrelation in digital holographic interferometry [11]-[12]. For that, a complex coherence factor between two speckled images from two digitally reconstructed holograms is of interest and described by:

$$
\mu=\frac{\left\langle A_{1} A_{2}^{*}\right\rangle}{\sqrt{\left\langle\left|A_{1}\right|^{2}\right\rangle\left\langle\left|A_{1}\right|^{2}\right\rangle}},
$$

where $A_{1}$ and $A_{2}$ are the two considered digital holographic images from the reconstruction algorithm (discrete Fresnel transform or angular spectrum transfer function). The coherence factor is linked to the standard deviation of speckle noise according to [13] (for $|\mu| \geq 0$.7):

$$
\sigma=\frac{7}{4}(1-|\mu|)^{2 / 5}
$$

and that is why modeling $|\mu|$ is of main interest in expected development of future model-based de-noising algorithms. The computation of Eq. (1) must consider the point spread function of digital holography [14] which includes the sensor area $\left(N p_{x}\right.$, with the $N$ number of pixels and the $p_{x}$ pixel pitch), the pixel surface width $(\Delta x)$, the wavelength of light $(\lambda)$ and the initial object-to-sensor distance $\left(d_{0}\right)$. Then, experimental parameters under study can be related to the spatial frequencies produced by the object in the particular case concerned. As an example of our recent results, in the case of surface deformation measurement (mechanical loads, vibrations, thermal loads, pneumatic loads), we demonstrate that $|\mu|$ has the expression in Eq. (3): 


$$
|\mu(u)|=\frac{\operatorname{sinc}(\pi \Delta x u) \operatorname{rect}\left(\frac{u-U_{x} / 2}{U_{x}}\right) \otimes \operatorname{sinc}(\pi \Delta x u) \operatorname{rect}\left(\frac{u-U_{x} / 2}{U_{x}}\right)}{\int_{0}^{U_{x}} \operatorname{sinc}(\pi \Delta x u) d u} .
$$

In Eq. (3), $U_{x}=N p_{\mathrm{x}} / \lambda d_{0}$ is the cut-off spatial frequency of Fresnel holography, $\operatorname{sinc}(x)=\sin (x) / x$ and $\operatorname{rect}(x)$ is the rectangle function. The same relation holds for the $y$ direction. The value of $|\mu|$ must be evaluated at the spatial frequency equivalent to the local slope of surface deformation [11]. Consequently, the speckle decorrelation noise is anisotropic and depends on both the dimensions of the sensor area and the local orientation of the fringes generated by the local surface slope of the deformation between the two holograms (refer to Fig. 1). This new result provides new opportunities for advanced de-noising methods in digital holographic metrology. Indeed, prior knowledge related to surface deformation could be used for predicting local noise and for processing adaptation. This would be very useful for high-speed holographic imaging because, in such a process, the number of pixels of the sensor is reduced and the decorrelation noise is stronger than in classical experiments. (a)

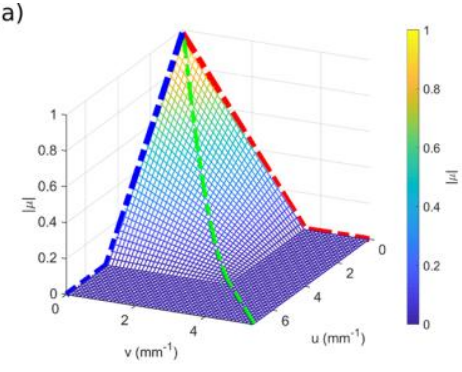

(c)

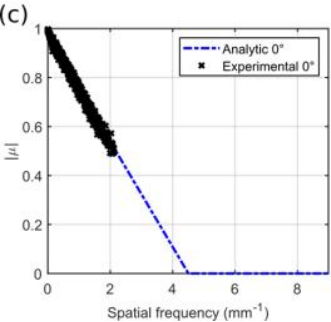

(b)

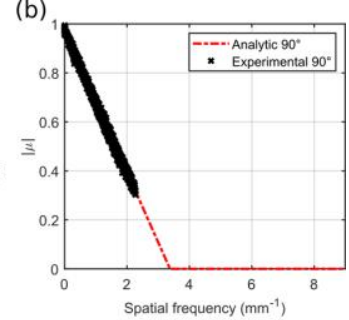

(d)

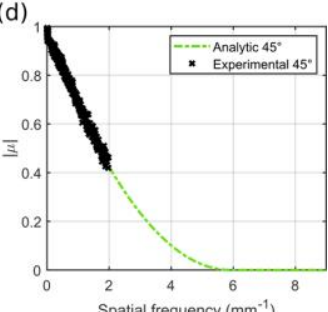

Fig. 1. Comparison between theory and experiments, (a) theoretical values of $|\mu|$, with the dashed blue line for the vertical fringes, the dashed red line for the horizontal orientation and the dashed green line for the inclined one, (b) comparison between experimental estimations of $|\mu|$ and theoretical values in the case of the horizontal fringes, (c) comparison between experimental estimations of $|\mu|$ and theoretical values in the case of the vertical fringes (d) comparison between experimental estimations of $|\mu|$ and theoretical values in the case of the $45^{\circ}$ fringes; from [11].

For the past 10 years, artificial intelligence (AI) and deep learning based on convolutional neural network have emerged as very efficient tools in signal and image processing with applications in speech and language understanding, or image recognition. It has now impacted digital holography for computer-generated holograms [10]. Soon, deep learning will probably strongly influence digital holography and its related post processing approaches. Especially, there is great interest in AI methods because they may strongly decrease the processing time for de-noising phase maps. Recently, we have demonstrated that the two-dimensional windowed Fourier transform (WFT2F) is the state-of-the art of speckle noise removal in speckle metrology [15] (refer to Fig. 2). The main drawback is a relatively long processing time to deal with phase maps including $1024 \times 1024$ data points.

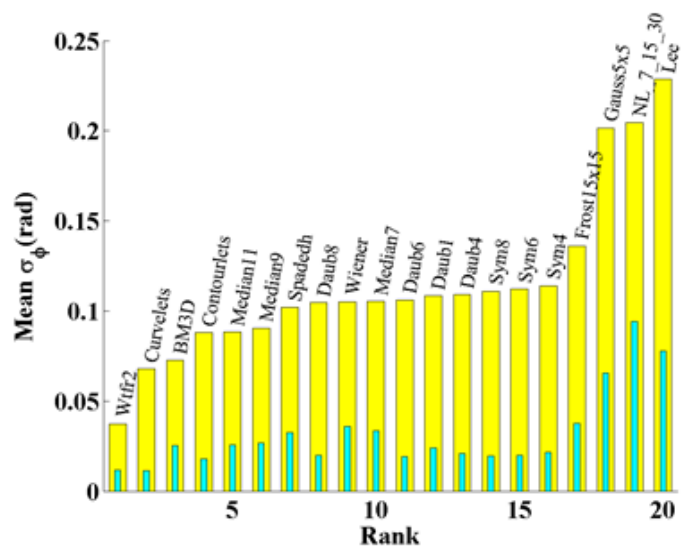

Fig. 2. Ranking of de-noising algorithms from the point of view of the standard deviation of the phase error; from [15].

To overcome such a limitation, we developed an approach based on deep learning [16]-[17]. One solution to improve de-noising with deep learning is to go deeper and to add more layers to the network. Despite higher capacity, overfitting and vanishing or exploding gradients arise as two main problems. The latter can be controlled by batch normalization and the use of skip connections such as in residual networks. But, to avoid overfitting even with regularization techniques, the amount of data is critical. Data augmentation generally helps in artificially increasing the amount of training data. Considering that a close relation does exist between the network depth and the size of convolutional filters (and the receptive field, consequently), the question of the depth requirement has not been thoroughly investigated.

The generalization power of machine learning algorithms is the "ability to perform well on previously unobserved inputs". To do so, data are usually split into training, development, and test sets, with the latter consisting of unobserved noisy phase data.

In our first approach [16], we trained a DnCNN (deep convolutional neural networks) for speckle de-noising in holographic phase data. The network reaches good performances with the benchmark data in comparison to other de-noising techniques such as BM3D, DTDWT or WFT2F. That was demonstrated for most of the evaluated phase data [16]. In our last developments, the aim is to reduce the training time while reaching similar performance. To do so, databases for development and 
validation were specifically designed and developed [17]. The main result is that a pre-trained model is not required unless the diversity and amount of simulated data are not consistent. In this case, the lack of data may be compensated by the pre-training. The experiments also demonstrated that very deep networks are not mandatory to use, and that four convolution blocks produce good performances when compared with WFT2F. Note that the advantage of reduced networks is the fact that they are fast to train. This study also addressed the issue of networks generalization. The paper [17] shows that the WFT2F algorithm continues to be the best one for phase images including strong noise (refer to Fig. 3). However, the baseline of WFT2F can be outperformed with our best model when considering experimental data. The deep learning approach was applied to experimental data from vibroacoustics based on on-line digital holography with a high-speed camera (refer to Fig. 4) and yielded excellent results compared to those provided by a scanning vibrometer [18].

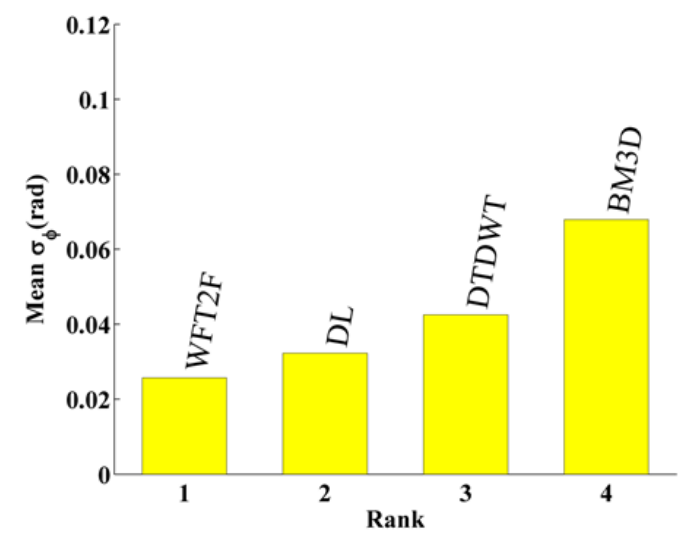

Fig. 3. Ranking of de-noising algorithms from the point of view of the standard deviation of the phase error; DL: our approach with deep learning; from [16].

In the future, we aim at investigating a multiplicative model. Improvement of speckle de-noising could be reached by combining the advantages of the two approaches and mixing in a noise estimator. To increase the amount of training data, we aim at implementing other data augmentation functions. Finally and additionally, a new database with an increased diversity of fringe images would be a matter of importance in learning the networks with a large diversity of phase fringe patterns.
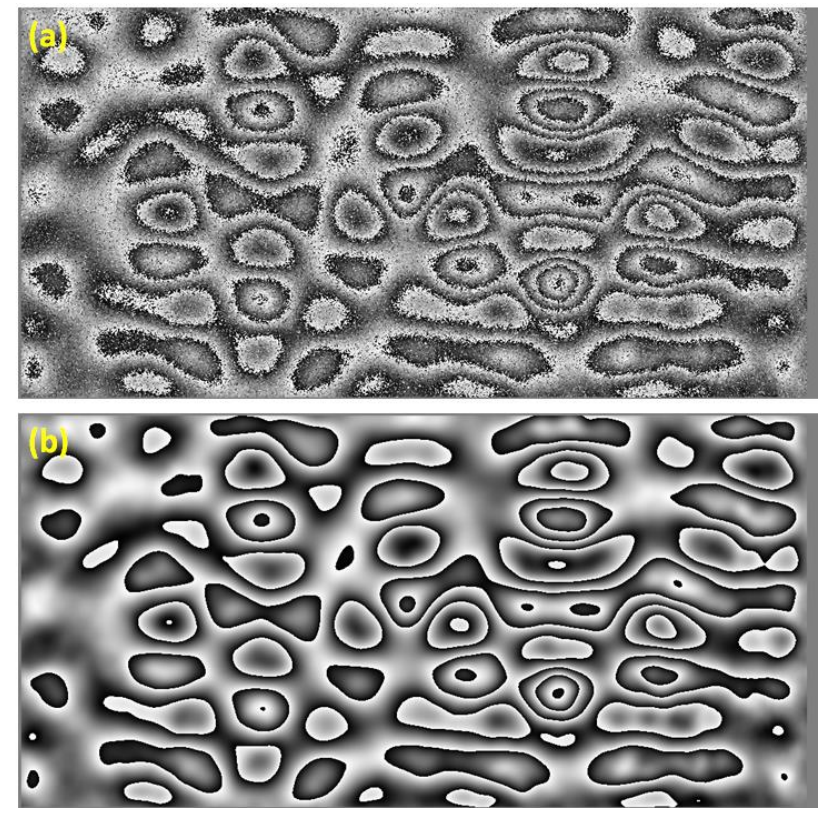

Fig. 4. (a) noisy phase map from the experiments in vibroacoustics, (b) de-noised phase processed from our deep learning approach; from [17].

\section{References}

[1] P. Picart (ed.), New techniques in digital holography (John Wiley \& Sons, 2015).

[2] T.M. Biewer, J.C. Sawyer, C.D. Smith, C.E. Thomas, Rev. Sci. Instr. 89, 10J123 (2018).

[3] M. Fratz, T. Beckmann, J. Anders, A. Bertz, M. Bayer, T. Gießler, C. Nemeth, D. Carl, Appl. Opt. 58(34), G120 (2019).

[4] M.P. Georges, J.-F. Vandenrijt, C. Thizy, Y. Stockman, P. Queeckers, F. Dubois, D. Doyle, Appl. Opt. 52(1), A102 (2013).

[5] E. Meteyer, F. Foucart, M. Secail-Geraud, P. Picart, C. Pezerat, Mech. Syst. Signal Process. 164 (2022).

[6] L. Lagny, M. Secail-Geraud, J. Le Meur, S. Montresor, K. Heggarty, C. Pezerat, P. Picart, J. Sound Vib. 461114925 (2019).

[7] L. Valzania, Y. Zhao, L. Rong, D. Wang, M. Georges, E. Hack, P. Zolliker, Appl. Opt. 58, G256 (2019).

[8] V. Bianco, P. Memmolo, M. Leo, S. Montresor, C. Distante, M. Paturzo, P. Picart, B. Javidi, P. Ferraro, Light: Sci. Appl. 7(1), 1 (2018).

[9] V. Bianco, P. Memmolo, M. Paturzo, A. Finizio, B. Javidi, P. Ferraro, Light. Sci. Appl. 5(9), e16142 (2016).

[10] R. Horisaki, R. Takagi, J. Tanida, Appl. Opt. 57(14), 3859 (2018).

[11] E. Meteyer, F. Foucart, C. Pezerat, P. Picart, Opt. Expr. 29(22), 36180 (2021).

[12] M. Piniard, B. Sorrente, G. Hug, P. Picart, Opt. Expr. 29(10), 14720 (2021).

[13] P. Picart, S. Montresor, O. Sakharuk, L. Muravsky, Opt. Lett. 42(2), 275 (2017).

[14] P. Picart, J. Leval, J. Opt. Soc. Am. A 25, 1744 (2008).

[15] S. Montresor, P. Picart, Opt. Expr. 24(13), 14322 (2016).

[16] S. Montresor, M. Tahon, A. Laurent, P. Picart, APL Photonics 5(3), 030802 (2020).

[17] M. Tahon, S. Montresor, P. Picart, Photonics 8(7), 255 (2021).

[18] E. Meteyer, S. Montresor, F. Foucart, J. Le Meur, K. Heggarty, C. Pezerat, P. Picart, Sci. Rep. 11(1), 1 (2021). 